\title{
¿CUÁL ES LA AGENDA DE DESARROLLO POS-2015? UNA MIRADA DESDE LOS DETERMINANTES DEL RIESGO DE DESASTRES
}

\author{
Juan Pablo Sarmiento P. ${ }^{1, *}$
}

\section{RESUMEN}

Este estudio explora el impacto actual y proyectado de las tres agendas de desarrollo pos-2015 en los determinantes del riesgo de desastres: el Marco de Sendai para la Reducción del Riesgo de Desastres, los Objetivos de Desarrollo Sostenible, y el Acuerdo de Paris COP21. La metodología se basa en un proceso ontológico, entendido éste como un análisis exhaustivo de las propiedades y relaciones de una entidad o sujeto de estudio, para caracterizar y vincular los objetivos propuestos en las tres agendas a través del impacto de los resultados esperados sobre los determinantes del riesgo de desastres. Se identificaron primero los elementos comunes al tema de riesgo de desastre de cada agenda y luego se empleó una serie de herramientas que siguen la propuesta de la Teoría de Cambio. Se identificaron trayectorias de cambio y con ello, momentos y áreas de intervención para actuar sobre las causas subyacentes del riesgo de desastre y contrarrestar así la construcción del riesgo. Es indispensable que exista una coherencia, complementariedad e interdependencia entre las tres agendas analizadas, de forma de trascender el tan anhelado y cuestionado crecimiento económico y así apuntalar un verdadero desarrollo sostenible, incidiendo sobre los factores determinantes del riesgo de desastre.

\section{PALABRAS CLAVE}

Riesgo, desarrollo sostenible, clima, Sendai, COP21

\begin{abstract}
This study explores the current and projected impacts of the three post 2015 development agendas on the determinants of disaster risk: the Sendai Framework for Disaster Risk Reduction, the Sustainable Development Goals, and the Paris COP21 Agreement. The methodology is based on an ontological process, understood as an exhaustive analysis of the properties and relationships of an entity or subject of study. The process characterized and linked the objectives proposed in the three agendas through the impact of the expected results on the determinants of disaster risk. First, elements related to disaster risk within each agenda were identified. Then, following the Theory of Change, a series of tools were used to identify domains of change, pathways, breakthroughs, and incremental outcomes that counteract the construction of disaster risk by acting on the underlying causes. It is essential that there be coherence, complementarity and interdependence between the three agendas analysed in order to transcend the longed for and questioned economic growth, and thus underpin true sustainable development by focusing on the determinants of disaster risk.
\end{abstract}

\section{KEYWORDS}

Risk, sustainable development, climate, Sendai, COP21
1. Profesor Investigador. Extreme Events Institute, Florida International University. $11200 \mathrm{SW}$ 8th Street, $\mathrm{AHC}_{5}$, Miami, FL 33199, EEUU.

*Autor de correspondencia: jsarmien@fiu.edu

\section{RECIBIDO}

31 de enero de 2017

\section{ACEPTADO}

20 de febrero de 2017

\section{PUBLICADO}

15 de julio de 2017

\section{Formato cita}

Recomendada (APA):

Sarmiento, J.P. (2017). ¿Cuál es la agenda de desarrollo pos-2015? Una mirada desde los determinantes del riesgo de desastres. REDER, 1(1), pp.29-42.

\section{(ब) $(1) \Theta$}

Todos los artículos publicados en REDER siguen una política de Acceso Abierto y se respaldan en una Licencia CreativeCommons Atribución-NoComercial 4.0 Internacional.

Revista de Estudios Latinoamericanos sobre Reducción del Riesgo de Desastres (REDER)

Diseño: Lupe Bezzina Tipografía: Hospital 


\section{INTRODUCCIÓN}

Durante el 2015 se concluyeron tres procesos de negociación para definir agendas de trabajo a nivel internacional en los temas de riesgo de desastres, desarrollo sostenible y cambio climático. Cada uno de estos temas estuvo precedido de meses, sino años, de cuidadosa preparación, que incluyó a técnicos y científicos, así como extensas negociaciones entre administradores públicos, economistas, legisladores y políticos.

Cada agenda debía culminar en un lugar y fecha en particular, y con un acto que plasmara la voluntad de los países firmantes y de los diferentes organismos de la comunidad internacional y la sociedad civil. El tema de Riesgos de Desastres debería concretarse en Sendai (Japón) durante la Conferencia Mundial para la Reducción de Riesgo de Desastres (RRD) convocada por Naciones Unidas entre el 14 y el 18 de marzo de 2015. El tópico de Desarrollo Sostenible fue finalmente preparado y adoptado durante la cumbre de las Naciones Unidas en la ciudad de Nueva York (Estados Unidos) en sesión plenaria de alto nivel de la Asamblea General, del 25 al 27 de septiembre de 2015. El tema de cambio climático se culminó en la XXI Conferencia Internacional sobre Cambio Climático - 21a Conferencia de las Partes y la $11^{\text {a }}$ Conferencia de las Partes en calidad de reunión de las Partes en el Protocolo de Kyoto (COP21/CMP11), celebrada en París (Francia), entre el 30 de noviembre y el 11 de diciembre de 2015 (Sarmiento, 2017).

Las tres agendas cuentan con grupos de interés específicos, prioridades y necesidades exclusivas, así como expectativas determinadas. Sin embargo, en su preparación se incluyeron esfuerzos particulares que buscaban sinergias, puntos de encuentro, evitando duplicidad e incluso inconsistencias entre las tres propuestas.

Desde el punto de vista del riesgo de desastres mucho se ha hablado de la confluencia de las tres agendas en busca de reducir la vulnerabilidad y construir resiliencia, reduciendo las pérdidas humanas y económicas de los desastres, protegiendo los medios de vida, reduciendo los niveles de pobreza y asegurando un desarrollo sostenible, tal como se expresa en la Figura 1 (Roberts et al. 2015).

Figura 1. Agenda de desarrollo pos-2015 Gráfico adaptado de Roberts et al. (2015).

Sin embargo, a la hora de entrar en la especificidad de cada agenda, no es fácil visualizar los puntos de encuentro, traslapes o diferencias que faciliten una toma de decisiones informadas a nivel de país y más aún en el nivel local. Se enfatiza en este estudio la compresión de las propuestas asociadas a la RRD de la agenda Pos-2015 a un nivel local, ya que es a este nivel donde se concreta y evidencia el riesgo de desastre.

\section{UNA MIRADA A LOS ACUERDOS QUE DEFINEN LA AGENDA POS-2015 DESDE LA PERSPECTIVA DEL RIESGO DE DESASTRE}

En esta sección se incluye una descripción concisa de cada agenda, extrayendo los elementos esenciales asociados con acciones concretas indicadas en los objetivos, así como en los principios rectores para el caso de agenda de gestión del riesgo de desastres, y las decisiones para hacer efectivo el acuerdo para la agenda climática. 


\subsection{Marco de Sendai para la Reducción del Riesgo de Desastres}

El Marco de Sendai para la Reducción del Riesgo de Desastres 2015-2030 (MSRRD) es el resultado de una serie de negociaciones que se iniciaron en marzo de 2012, y que culminaron el 18 de marzo de 2015 durante la Conferencia Mundial para la RRD en Sendai (Japón) donde fue adoptado por 187 naciones. Si bien el marco fue aprobado en la fecha mencionada, fue necesario que un grupo de trabajo intergubernamental y de expertos liderado por la Oficina de las Naciones Unidas para la Reducción del Riesgo de Desastres (UNISDR por las siglas en inglés) trabajara en dos temas que quedaron pendientes en Sendai: 1) los indicadores globales para medir el progreso en el logro de los objetivos del MSRRD, y 2) la actualización de terminología de Naciones Unidas sobre reducción del riesgo, cuya última versión era de 2009. Los resultados de este grupo de trabajo no fueron divulgados sino hasta diciembre de 2016 (Naciones Unidas, 2016).

Es importante resaltar ciertos antecedentes del MSRRD. El Marco de Sendai es antecedido por el Marco de Acción de Hyogo cuya vigencia fue del 2005 al 2015 y cuyo énfasis estuvo en el aumento de la resiliencia ante los desastres. El Marco de Acción de Hyogo buscaba a su vez consolidar la acción del llamado Marco Internacional de Acción del Decenio Internacional para la Reducción de los Desastres Naturales de 1989 y la Estrategia de Yokohama de 1994. La década de los 90s fue la primera acción decisiva a nivel internacional para promover un cambio de paradigma: del manejo de emergencias y desastres a la RRD.

La Tabla 1 resume los principales componentes del MSRRD:

\section{Alcance y objetivo}

El presente Marco se aplicará al riesgo de desastres de pequeña y gran escala, frecuentes y poco frecuentes, súbitos y de evolución lenta, naturales o causados por el hombre, así como a las amenazas y los riesgos ambientales, tecnológicos y biológicos conexos. Tiene por objeto orientar la gestión del riesgo de desastres en relación con amenazas múltiples en el desarrollo a todos los niveles, así como en todos los sectores y entre un sector y otro.

\section{Resultado esperado}

La reducción sustancial del riesgo de desastres y de las pérdidas ocasionadas por los desastres, tanto en vidas, medios de subsistencia y salud como en bienes económicos, físicos, sociales, culturales y ambientales de las personas, las empresas, las comunidades y los países.

\section{Metas}

Prevenir la aparición de nuevos riesgos de desastres y reducir los existentes implementando medidas integradas e inclusivas de índole económica, estructural, jurídica, social, sanitaria, cultural, educativa, ambiental, tecnológica, política e institucional que prevengan y reduzcan la exposición a las amenazas y la vulnerabilidad a los desastres, aumenten la preparación para la respuesta y la recuperación, y de ese modo refuercen la resiliencia

\section{Prioridades de acción}

Los Estados deben adoptar medidas específicas en todos los sectores, en los planos local, nacional, regional y mundial, con respecto a las siguientes cuatro esferas prioritarias

\begin{tabular}{c|c|c|c}
$\begin{array}{c}\text { Comprender el riesgo de } \\
\text { desastres }\end{array}$ & $\begin{array}{c}\text { Fortalecer la gobernanza } \\
\text { del riesgo de desastres } \\
\text { para gestionar dicho riesgo }\end{array}$ & $\begin{array}{c}\text { Invertir en la reducción del } \\
\text { riesgo de desastres para la } \\
\text { resiliencia }\end{array}$ & $\begin{array}{c}\text { Aumentar la preparación } \\
\text { para casos de desastre a } \\
\text { fin de dar una respuesta } \\
\text { eficaz, y "reconstruir } \\
\text { mejor" en el ámbito de la } \\
\text { recuperación, la rehabili- } \\
\text { tación y la reconstrucción }\end{array}$ \\
\hline
\end{tabular}

\section{Principios rectores}

PR1. Los Estados tienen la responsabilidad primordial de prevenir y reducir el riesgo de desastres, entre otras cosas mediante la cooperación

PR2. Responsabilidad compartida entre los gobiernos centrales y las autoridades, los sectores y las partes interesadas a nivel nacional, según se considere adecuado en función de sus circunstancias nacionales

PR3. Proteger a las personas y sus bienes, al tiempo que se promueven y se protegen todos los derechos humanos, incluido el derecho al desarrollo

PR4. Colaboración de toda la sociedad

PR5. La plena participación de todas las instituciones ejecutivas y legislativas del Estado a nivel nacional y local

PR6. Se debe empoderar a las autoridades y las comunidades locales mediante recursos, incentivos y responsabilidades en materia de adopción de decisiones, según corresponda

PR7. La adopción de decisiones debe ser inclusiva y basada en el conocimiento sobre los riesgos, con un enfoque basado en peligros múltiples

PR8. Coherencia de las políticas, los planes, las prácticas y los mecanismos de reducción del riesgo de desastres y de desarrollo sostenible en los diferentes sectores

(continúa en la siguiente página) 
Principios rectores

PR9. Considerar las características locales y específicas de los riesgos de desastres a la hora de determinar las medidas para reducir el riesgo

PR10. Abordar los factores subyacentes del riesgo de desastres de manera rentable, realizando inversiones en lugar de depender principalmente de la respuesta y la recuperación después de un desastre

PR11. Una "reconstrucción mejor" para prevenir nuevos desastres y reducir el riesgo de desastres

PR12. La alianza mundial y la cooperación internacional deben ser efectivas, significativas y fuertes

PR13. El apoyo de los países desarrollados y asociados para los países en desarrollo debe adaptarse a sus necesidades y prioridades, según definan ellos mismos

Tabla 1. Marco de Sendai para la Reducción del Riesgo de Desastres 2015-2030

A continuación la tabla 2 contiene los 7 objetivos del MSRRD

Objetivos del Marco de Sendai para la Reducción del Riesgo de Desastres

OBJETIVO 1. Reducir considerablemente la mortalidad mundial causada por los desastres para 2030, y lograr reducir la tasa de mortalidad mundial por cada 100.000 personas en la década de 2020-2030 respecto del período 2005-2015

OBJETIVO 2. Reducir considerablemente el número de personas afectadas a nivel mundial para 2030, y lograr reducir el promedio mundial por cada 100.000 personas en la década 2020-2030 respecto del período 2005-2015

OBJETIVO 3. Reducir las pérdidas económicas causadas directamente por los desastres en relación con el producto interno bruto (PIB) mundial para 2030

OBJETIVO 4. Reducir considerablemente los daños causados por los desastres en las infraestructuras vitales y la interrupción de los servicios básicos, como las instalaciones de salud y educativas, incluso desarrollando su resiliencia para 2030

OBJETIVO 5. Incrementar considerablemente el número de países que cuentan con estrategias de reducción del riesgo de desastres a nivel nacional y local para 2020

OBJETIVO 6. Mejorar considerablemente la cooperación internacional para los países en desarrollo mediante un apoyo adecuado y sostenible que complemente las medidas adoptadas a nivel nacional para la aplicación del presente Marco para 2030

OBJETIVO 7. Aumentar considerablemente la disponibilidad y el acceso de las personas a los sistemas de alerta tempra na de peligros múltiples y a la información sobre el riesgo de desastres y las evaluaciones para el año 2030.

Tabla 2. Objetivos del Marco de Sendai para la Reducción del Riesgo de Desastres

Un reciente documento de UNISDR (2015) diseñado para interpretar el MSRRD enfatiza la diferencia entre el propósito, el resultado esperado y las metas acordadas. La finalidad del Marco entendida como la razón de ser de la RRD, la motivación y la intención de abordar las causas de fondo del riesgo de desastres y, por lo tanto, hacer el desarrollo sostenible. Esto implica que si el desarrollo contiene condiciones de riesgo no puede ser considerado desarrollo sostenible. El resultado esperado se refiere al cambio, al impacto que se espera generen las políticas, programas y acciones propuestos. Las metas buscan evitar la generación de nuevos riesgos, reducir el riesgo existente y fortalecer la resiliencia.

\subsection{Los Objetivos de Desarrollo Sostenible}

Los Objetivos de Desarrollo Sostenible (ODS) constituyen un imperativo universal dirigido a la adopción de medidas para poner fin a la pobreza, proteger el planeta y asegurar que todas las personas puedan disfrutar condiciones de paz y prosperidad. Los ODS amplían en forma importante a sus predecesores, los llamados Objetivos de Desarrollo del Milenio (ODM), pasando de ocho (ODM) objetivos y 21 metas, a 17 objetivos (ODS) y 169 metas. Una característica importante de los nuevos objetivos es que éstos están frecuentemente interrelacionados, lo cual implica un principio de complementariedad e interdependencia.

La tabla 3 detalla los 17 ODS formulados:

\section{Objetivos de Desarrollo Sostenible}

OBJETIVO 1. Poner fin a la pobreza en todas sus formas en todo el mundo

OBJETIVO 2. Poner fin al hambre, lograr la seguridad alimentaria y la mejora de la nutrición y promover la agricultura sostenible

OBJETIVO 3. Garantizar una vida sana y promover el bienestar para todos en todas las edades

OBJETIVO 4. Garantizar una educación inclusiva, equitativa y de calidad y promover oportunidades de aprendizaje du rante toda la vida para todos 


\section{Objetivos de Desarrollo Sostenible}

OBJETIVO 5. Lograr la igualdad entre los géneros y empoderar a todas las mujeres y las niñas

OBJETIVO 6. Garantizar la disponibilidad de agua y su gestión sostenible y el saneamiento para todos

OBJETIVO 7. Garantizar el acceso a una energía asequible, segura, sostenible y moderna para todos

OBJETIVO 8. Promover el crecimiento económico sostenido, inclusivo y sostenible, el empleo pleno y productivo y el trabajo decente para todos

OBJETIVO 9. Construir infraestructuras resilientes, promover la industrialización inclusiva y sostenible y fomentar la innovación

OBJETIVO 10. Reducir la desigualdad en y entre los países

OBJETIVO 11. Lograr que las ciudades y los asentamientos humanos sean inclusivos, seguros, resilientes y sostenibles OBJETIVO 12. Garantizar modalidades de consumo y producción sostenibles

OBJETIVO 13. Adoptar medidas urgentes para combatir el cambio climático y sus efectos

OBJETIVO 14. Conservar y utilizar en forma sostenible los océanos, los mares y los recursos marinos para el desarrollo sostenible

OBJETIVO 15. Proteger, restablecer y promover el uso sostenible de los ecosistemas terrestres, gestionar los bosques de forma sostenible, luchar contra la desertificación, detener e invertir la degradación de las tierras y poner freno a la pérdida de la diversidad biológica

OBJETIVO 16. Promover sociedades pacíficas e inclusivas para el desarrollo sostenible, facilitar el acceso a la justicia para todos y crear instituciones eficaces, responsables e inclusivas a todos los niveles

OBJETIVO 17. Fortalecer los medios de ejecución y revitalizar la Alianza Mundial para el Desarrollo Sostenible

Tabla 3. Objetivos de Desarrollo Sostenible

Los objetivos de desarrollo sostenible se diferencian significativamente de sus antecesores los objetivos del milenio. Las principales diferencias radican en que los objetivos del milenio se desarrollaron para los países en desarrollo desde un enfoque del donante, mientras los objetivos de desarrollo sostenible aplican a todos los países. Los objetivos de desarrollo sostenible van más allá de los síntomas de la pobreza y abordan temas como cambio climático, desigualdad económica, innovación, consumo sostenible, paz y justicia, derechos humanos y gobernanza. Adicionalmente, los objetivos de desarrollo sostenible abordan directamente temas cruciales como género, participación popular, y gobierno "a todos los niveles". Finalmente cabe mencionar la innovación al incluir el tema de información, indicando la importancia de la disponibilidad y acceso a datos (demografía, economía, etc.) de alta calidad en los contextos nacionales.

\subsection{El Acuerdo de París 2015}

El objetivo central del Acuerdo de París es fortalecer la respuesta mundial a la amenaza del cambio climático, manteniendo el aumento de la temperatura global de este siglo muy por debajo de los 2 grados centígrados (por encima de los niveles preindustriales), y proseguir los esfuerzos para limitar el aumento de la temperatura a 1,5 grados Celsius. Además, el acuerdo tiene como objetivo fortalecer la capacidad de los países para hacer frente a los impactos del cambio climático. Para alcanzar estos ambiciosos objetivos, se establecerán flujos financieros apropiados, un nuevo marco tecnológico y un marco mejorado de creación de capacidades, apoyando así la acción de los países en desarrollo y los países más vulnerables, de conformidad con sus propios objetivos nacionales. El Acuerdo también prevé un marco de transparencia más sólido para fortalecer el proceso de rendición de cuentas y la confianza en la acción.

La tabla 4 recoge las principales características del Acuerdo de París.

\begin{tabular}{|c|c|}
\hline \multicolumn{2}{|r|}{ Acuerdo de París COP21 } \\
\hline PROPÓSITO & $\begin{array}{l}\text { Reforzar la respuesta mundial a la amenaza del cambio climático, en el contexto del } \\
\text { desarrollo sostenible y de los esfuerzos por erradicar la pobreza. }\end{array}$ \\
\hline OBJETIVOS A LARGO PLAZO & $\begin{array}{l}\text { 1. Mantener el aumento de la temperatura media mundial muy por debajo de } 2^{\circ} \mathrm{C} \text { con } \\
\text { respecto a los niveles preindustriales, y proseguir los esfuerzos para limitar ese aumento } \\
\text { de la temperatura a } 1,5^{\circ} \mathrm{C} \text { con respecto a los niveles preindustriales, reconociendo que } \\
\text { ello reduciría considerablemente los riesgos y los efectos del cambio climático; } \\
\text { 2. Aumentar la capacidad de adaptación a los efectos adversos del cambio climático y } \\
\text { promover la resiliencia al clima y un desarrollo con bajas emisiones de gases de efecto } \\
\text { invernadero, de un modo que no comprometa la producción de alimentos; } \\
\text { 3. Elevar las corrientes financieras a un nivel compatible con una trayectoria que } \\
\text { conduzca a un desarrollo resiliente al clima y con bajas emisiones de gases de efecto } \\
\text { invernadero. }\end{array}$ \\
\hline
\end{tabular}


Decisiones para hacer efectivo el acuerdo

D1. MITIGACIÓN

- Mantener el aumento de la temperatura media mundial muy por debajo de los $2^{\circ} \mathrm{C}$ por encima de los niveles preindustriales; Procurar limitar el aumento a $1,5^{\circ} \mathrm{C}$, ya que esto reduciría significativamente los riesgos y los impactos del cambio climático;

- Reducir las emisiones mundiales en un máximo lo antes posible, reconociendo que esto requerirá más tiempo para los países en desarrollo.

- Realizar rápidas reducciones a partir de entonces de acuerdo con la mejor ciencia disponible D2. ADAPTACIÓN — - Mejorar la capacidad de adaptación, fortalecer la resiliencia y reducir la vulnerabilidad de las sociedades para enfrentar los efectos del cambio climático.

- Proporcionar un apoyo internacional continuado para la adaptación al cambio climático de los países en desarrollo.

- Reportar el progreso en adaptación, incluyendo la adecuación y eficacia de las medidas y del apoyo recibido, cada cinco años.

D3. PÉRDIDAS Y DAÑOS _ - Reconoce la importancia de evitar, minimizar y abordar las pérdidas y los daños aso ciados con los efectos adversos del cambio climático (incluidos los fenómenos meteorológicos extremos y los eventos de inicio lento, como el aumento del nivel del mar).

- Reconoce la necesidad de cooperar y mejorar la comprensión, la acción y el apoyo en diferentes ámbitos, como los sistemas de alerta temprana, la preparación para casos de emergencia y el seguro contra riesgos.

- Nota. Lo anterior no implica ni proporciona una base para ninguna responsabilidad o compensación

D4. TRANSPARENCIA Y — - Reunirse cada 5 años para establecer nuevos y más ambiciosos objetivos, basados en VALORACIÓN GLOBAL_ evidencia científica.

- Seguimiento del progreso hacia el objetivo a largo plazo a través de un sólido sistema de transparencia y rendición de cuentas. Los países deben informar públicamente sobre el avance en la implementación de los objetivos.

- El nuevo sistema de transparencia se basa en compromisos vinculantes comunes para todas las partes y en una "flexibilidad integrada" para adaptarse a las diferentes capa cidades nacionales.

D5. MERCADOS DE CARBONO - Las Partes pueden utilizar "resultados de mitigación internacionalmente transferidos" para implementar sus NDC, evitando el doble conteo.

D6. FINANCIACIÓN — - Se fijó una meta de 100.000 millones de dólares al año hasta el año 2025

- El tema de finanzas fue controvertible en París, con claras diferencias entre países en desarrollo, particularmente los más pobres, los países desarrollados y aquellos países en desarrollo más ricos

Este cuadro fue preparado con base en el Acuerdo de Paris (United Nations, 2015), el reporte del C2ES sobre los resultados de la Conferencia de Paris (C2ES, 2015), y el resumen de Corbyn (2016) del Servicio de Investigación de Gales.

Tabla 4. Acuerdo de París COP21

El acuerdo de Paris deja claro que aun cuando hay confianza en que el riesgo global del cambio climático puede reducirse limitando la tasa y la magnitud de las emisiones y manteniendo el aumento de la temperatura global, hay un hecho indiscutible, que el riesgo de desastres está tan estrechamente ligado a la exposición y la vulnerabilidad, que es necesario enfatizar que tanto la adaptación como la reducción de riesgos deben entenderse en el contexto de un desarrollo social y económico más amplio.

\section{OBJETIVO Y METODOLOGÍA}

\subsection{Objetivo del estudio}

Explorar el impacto actual y proyectado de las tres agendas de desarrollo pos-2015 en los determinantes del riesgo de desastres.

\subsection{Metodología}

El estudio empleó un proceso ontológico, entendido como un análisis exhaustivo de las propiedades y relaciones de una entidad o sujeto de estudio (Zalta, 2009), para caracterizar y vincular los objetivos propuestos en las tres agendas: desarrollo sostenible, cambio climático y riesgo de desastres, a través de escenarios y del impacto de los resultados esperados sobre los determinantes del riesgo de desastres, siguiendo la propuesta metodológica de Goldhagen y Mercer (2010).

Se identificaron primero los elementos comunes al tema de riesgo de desastre de cada agenda, luego empleando un conjunto de herramientas se analizó y abordó su impacto actual y proyectado sobre los determinantes del riesgo de desastres (tablas 5,6 y 7; figuras 3 y 4). Estos análisis de propiedades y relaciones siguen la propuesta de la Teoría de Cambio (Weiss, 1998), la cual describe un proceso de cambio social deseado haciendo explícito la manera en que pensamos sobre un problema actual, sus causas subyacentes, el cambio a largo plazo que buscamos y lo que deber 
ocurrir en la sociedad para que ese cambio surja. La Teoría de Cambio es entonces un vínculo entre la teoría y la acción, basados en hipótesis que sustentan nuestros pensamientos y creencias sobre lo que mejorará la resiliencia ante los desastres y por qué (TANGO International, 2012).

Aun cuando cada una de las herramientas mencionadas puede utilizarse individualmente 0 en combinación, el hecho de emplearlas secuencialmente ofrece un proceso lógico y coherente, y que como indican Goldhagen y Mercer (2010), puede tener varios propósitos, desde educar hasta informar a tomadores de decisiones.

\subsection{Marco Conceptual}

El presente estudio se construye sobre una interpretación del modelo de presión y liberación propuesto por Blaikie et al. (1994) ya que nos permite relacionar las condiciones de riesgos a partir de la compleja relación entre las amenazas y la vulnerabilidad (Figura 2), entendida ésta última como el resultado de una progresión que se origina en unas causas de fondo (Procesos ideológicos de carácter económico, demográfico y político que influyen en las relaciones de poder y la asignación y distribución de recursos en una sociedad), las cuales se manifiestan en unas presiones dinámicas (crecimiento de la población, migración, urbanización acelerada, etc.), que a su vez resultan y se visualizan como condiciones inseguras (segmentación de la sociedad, medios de vida inestables, ocupación del territorio con exposición a amenazas, manejo inadecuado de emergencias).

$\begin{gathered}\text { Causas } \\ \text { de fondo }\end{gathered} \rightarrow \begin{gathered}\text { Presiones } \\ \text { dinámicas }\end{gathered} \rightarrow \begin{gathered}\text { Condiciones } \\ \text { inseguras }\end{gathered} \rightarrow \begin{gathered}\text { Riesgo }=\underset{\text { Vulnerabilidad }}{f \text { Exposicin, Amenaza y }} \\ \text { Vulneras }\end{gathered}-$ Amenazas

Figura 2. Modelo de Presión y Liberación de los Desastres

Adaptado del modelo de presión y liberación propuesto por Blaikie et al. (1994)

Interesante recalcar en este modelo el carácter dinámico y de permanente cambio de los procesos involucrados. En este marco y para este estudio, se adoptan cuatro conceptos básicos que han sido revisados recientemente por Naciones Unidas, a saber:

\section{Amenaza}

"Proceso, fenómeno o actividad humana que puede ocasionar muertes, lesiones u otros efectos en la salud, daños a los bienes, disrupciones sociales y económicas o daños ambientales" (Naciones Unidas, 2016, p.19)

\section{Vulnerabilidad}

“Condiciones determinadas por factores o procesos físicos, sociales, económicos y ambientales que aumentan la susceptibilidad de una persona, una comunidad, los bienes o los sistemas a los efectos de las amenazas" (Naciones Unidas, 2016, p.25).

\section{Resiliencia}

"Capacidad que tiene un sistema, una comunidad o una sociedad expuestos a una amenaza para resistir, absorber, adaptarse, transformarse y recuperarse de sus efectos de manera oportuna y eficiente, en particular mediante la preservación y la restauración de sus estructuras y funciones básicas por conducto de la gestión de riesgos" (Naciones Unidas, 2016, p.23).

\section{Factores subyacentes del riesgo de desastres}

"Procesos o condiciones, a menudo relacionados con el desarrollo, que influyen en el nivel de riesgo de desastres al incrementar los niveles de exposición y vulnerabilidad o reducir la capacidad" (Naciones Unidas, 2016, p.25).

Para el presente estudio el autor considera como sinónimos los determinantes del riesgo y los factores subyacentes del riesgo, e igualmente afirma que éstos engloban los tres procesos descritos por Blaikie y sus coautores (1994) en la progresión donde hay unas causas de fondo que resultan en presiones dinámicas y se manifiestan en condiciones de inseguridad. 


\section{RESULTADOS Y DISCUSIÓN}

Siguiendo la metodología propuesta se presentan a continuación los resultados del análisis de propiedades y relaciones de las tres agendas estudiadas, mediante el uso de diferentes herramientas como la matriz de gestión del riesgo, el árbol de problemas, el mapa de cambio, y la matriz de dominios intervención sobre los determinantes del riesgo de desastres.

\subsection{Matriz de gestión del riesgo}

Esta herramienta permite identificar y organizar los elementos comunes al tema de riesgo de desastre de cada agenda, para luego analizar y abordar su impacto actual y proyectado sobre los determinantes del riesgo de desastres (Tabla 5).

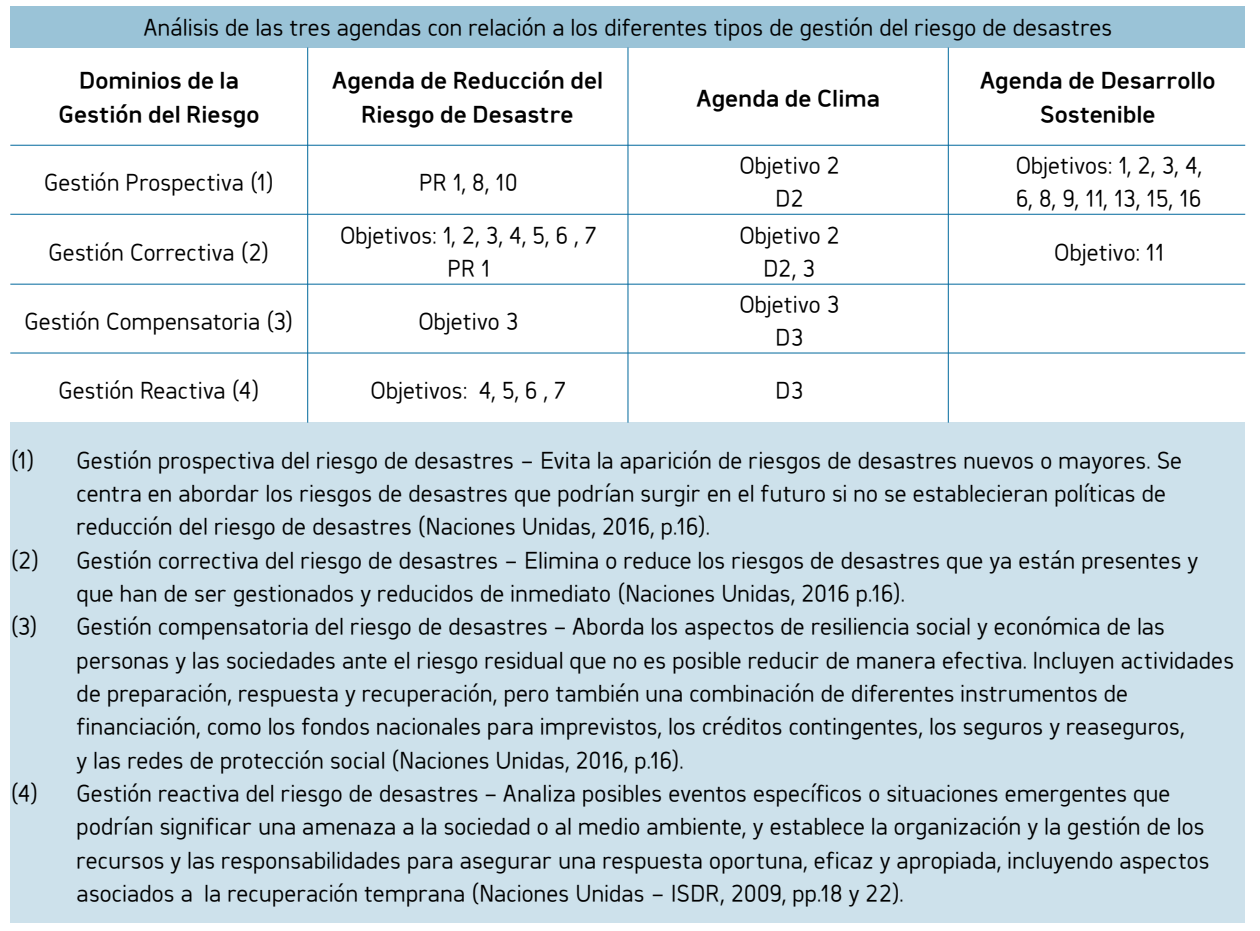

Tabla 5. Análisis de las tres agendas con relación a los diferentes tipos de gestión del riesgo de desastres

Este ejercicio muestra con claridad que la gestión prospectiva del riesgo está siendo abordada principalmente por la agenda de desarrollo sostenible, mientras que la gestión correctiva, compensatoria y reactiva se encuentra cubierta por la agenda de reducción de riesgos. La agenda de clima, muestra un interesante balance en los cuatro tipos de gestión.

\section{2 Árbol del Problema}

Esta herramienta es esencial para el análisis ontológico propuesto. La preparación del árbol del problema aprovechó y amplió el marco conceptual propuesto por Blaikie (1994). El análisis se centró en la problemática de la construcción del riesgo de desastre, entendida como "la producción y reproducción de las condiciones de vulnerabilidad que definen y determinan la magnitud de los efectos ante la presencia de una amenaza natural; es por ello la principal responsable de los procesos de desastre." (Acosta, 2005, p.23)

Declaración del problema: Construcción del riesgo de desastre en una sociedad en particular (incluye población, territorio, infraestructura, bienes, y servicios) que definen y determinan la magnitud de los efectos ante la presencia de una(s) amenaza(s) específica(s). Esta declaración identifica el problema y el contexto en el cual se gesta (McGaghie et al. 2001) indicando claramente el 'qué' (problema: inconveniente, dificultad o diferencia entre lo que existe y lo que debiera ser), el 'quién' (el sujeto afectado por el problema) y el 'dónde' (ubicación geográfica).

A continuación, empleando el árbol del problema (Figura 3) se procedió a identificar las llamadas causas subyacentes de la problemática, que también se ha llamado en este estudio factores determinantes o subyacentes del riesgo, representados por las raíces, identificando luego causas principales y secundarias, resultando en un proceso de concatenación y jerarquización que llega hasta las manifestaciones visibles de la problemática analizada. 


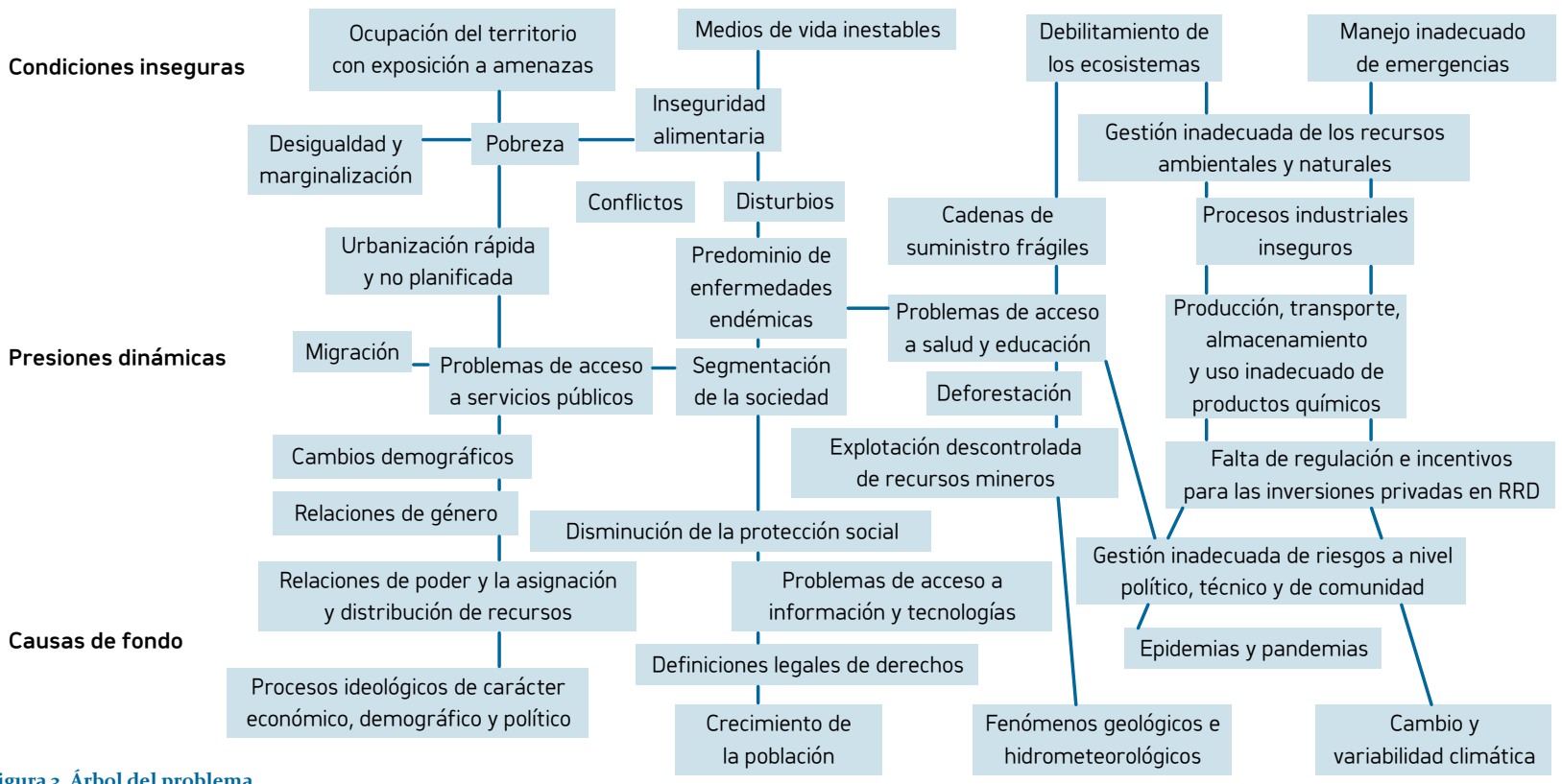

Al organizar los datos se procedió a identificar los puntos clave de influencia, entendidos como el (los) camino(s) de cambio porque son repetidamente identificados entre numerosos flujos causales. Estos caminos de cambio identifican trayectorias entre los dominios o procesos descritos por Blaikie et al. (1994), complementados por el trabajo que llevó a cabo el grupo actualización de terminología del Naciones Unidas (2016) en cuanto a factores subyacentes.

\subsection{Mapa de Cambio}

Los dominios de cambio o puntos clave de influencia, en este estudio son llamados dominios de intervención, áreas principales en las cuales el cambio debe ocurrir para poder alcanzar el objetivo de impacto deseado. Para este estudio el objetivo a alcanzar fue definido como: 'Desarrollo sostenible y seguro para una sociedad en particular'.

La Figura 4 registra el mapa de cambio obtenido a partir del árbol del problema identificado, el objetivo de impacto deseado y las trayectorias resultantes entre estos.

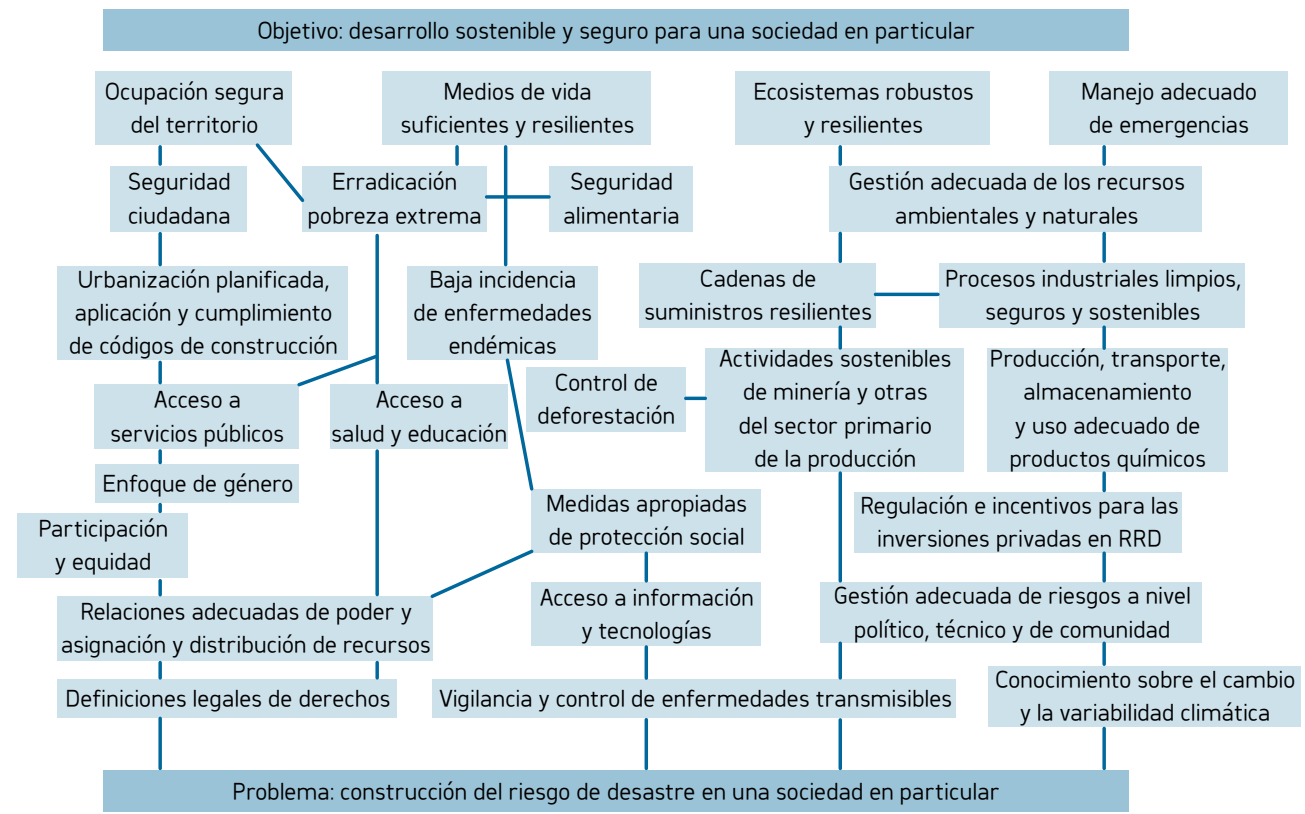


Como puede observarse en la Figura 4, hay cuatro dominios principales que se deberían abordar para alcanzar el objetivo deseado, representados en el gráfico en la línea de condiciones inseguras.

- Ocupación segura del territorio

- Medios de vida suficientes y resilientes

- Ecosistemas robustos y resilientes

- Manejo adecuado de emergencias

Es de anotar que hay un nodo de particular importancia que debe ser abocado: erradicación de pobreza absoluta. Este influye en dos de los cuatro dominios principales.

Estos dominios se obtuvieron a partir de las causas subyacentes del problema, las cuales fueron convertidas en resultados incrementales y puntos de avance que comprenden caminos de cambio necesarios para alcanzar el objetivo de impacto propuesto. Es importante resaltar que no todas las causas subyacentes fueron transformadas en resultados incrementales. En su selección se emplearon criterios pertinencia, relevancia y particularmente la factibilidad de llevarlas a cabo.

\subsection{Matriz de dominios de intervención}

Las trayectorias resultantes constituyen a la vez caminos de influencia sobre los cuales se puede (debe) actuar para incidir en los determinantes del riesgo de desastres. La actuación se hace a través de intervenciones específicas, que en este estudio corresponde a las propuestas contenidas en las tres agendas de desarrollo pos-2015. La tabla 6 muestra la matriz resultante del análisis de los dominios de intervención y las propuestas de las tres agendas.

\begin{tabular}{|c|c|c|c|}
\hline \multicolumn{4}{|c|}{ Matriz de intervención sobre los determinantes del riesgo de desastres } \\
\hline Intervenciones & $\begin{array}{l}\text { Agenda de Reducción del } \\
\text { Riesgo de Desastre }\end{array}$ & Agenda de Clima & $\begin{array}{c}\text { Agenda de Desarrollo } \\
\text { Sostenible }\end{array}$ \\
\hline $\begin{array}{l}\text { Dominio 1: Ocupación } \\
\text { segura del territorio }\end{array}$ & PR 10 & & Objetivo 11 \\
\hline Seguridad ciudadana & & & Objetivo 16 \\
\hline $\begin{array}{l}\text { Urbanización planificada, } \\
\text { aplicación y cumplimiento } \\
\text { de códigos de construcción }\end{array}$ & & D2 & Objetivo 9 \\
\hline Acceso a servicios públicos & Objetivo 4 & & Objetivos 6, 7 \\
\hline Enfoque de género & & & Objetivo 5 \\
\hline Participación y equidad & PR4, PR5, PR6, PR7 & & Objetivos $10,11,16$ \\
\hline $\begin{array}{l}\text { Relaciones adecuadas de } \\
\text { poder y asignación y } \\
\text { distribución de recursos }\end{array}$ & & & Objetivos 10, 11, 16 \\
\hline $\begin{array}{l}\text { Definiciones legales } \\
\text { de derechos }\end{array}$ & PR3 & & Objetivos $10,11,16$ \\
\hline $\begin{array}{l}\text { Dominio 2: Medios de vida } \\
\text { suficientes y resilientes }\end{array}$ & PR10 & & Objetivo 8 \\
\hline $\begin{array}{c}\text { Erradicación pobreza } \\
\text { extrema }\end{array}$ & PR3 & & Objetivo 1 \\
\hline Seguridad alimentaria & & Objetivo 2 & Objetivo 2 \\
\hline \multicolumn{4}{|l|}{$\begin{array}{c}\text { Baja incidencia de } \\
\text { enfermedades endémicas }\end{array}$} \\
\hline Acceso a salud y educación & Objetivo 4 & & Objetivos 3,4 \\
\hline $\begin{array}{c}\text { Cadenas de } \\
\text { suministro resilientes }\end{array}$ & & & Objetivo 12 \\
\hline $\begin{array}{l}\text { Medidas apropiadas } \\
\text { de protección social }\end{array}$ & & & Objetivo 8 \\
\hline $\begin{array}{c}\text { Acceso a información } \\
\text { y tecnologías }\end{array}$ & & & \\
\hline
\end{tabular}

(continúa en la siguiente página) 


\begin{tabular}{|c|c|c|c|}
\hline \multicolumn{4}{|c|}{ Matriz de intervención sobre los determinantes del riesgo de desastres } \\
\hline Intervenciones & $\begin{array}{c}\text { Agenda de Reducción del } \\
\text { Riesgo de Desastre }\end{array}$ & Agenda de Clima & $\begin{array}{c}\text { Agenda de Desarrollo } \\
\text { Sostenible }\end{array}$ \\
\hline $\begin{array}{l}\text { Dominio 3: Ecosistemas } \\
\text { robustos y resilientes }\end{array}$ & PR10 & & Objetivo 15 \\
\hline $\begin{array}{c}\text { Gestión adecuada de los } \\
\text { recursos ambientales } \\
\text { y naturales }\end{array}$ & & D2 & Objetivo 15 \\
\hline $\begin{array}{c}\text { Procesos industriales } \\
\text { limpios, seguros y sostenibles }\end{array}$ & & Objetivo 1 - D1 & Objetivo 9 \\
\hline Control de Deforestación & & $\mathrm{D} 2$ & Objetivo 15 \\
\hline $\begin{array}{l}\text { Actividades sostenibles de } \\
\text { minería y otras del sector } \\
\text { primario de la producción }\end{array}$ & & D2 & Objetivo 14 \\
\hline $\begin{array}{l}\text { Regulación e incentivos } \\
\text { para las inversiones } \\
\text { privadas en RRD }\end{array}$ & & D2, D3 & \\
\hline $\begin{array}{l}\text { Dominio 4: Manejo ade- } \\
\text { cuado de emergencias }\end{array}$ & Objetivos 1, 2, 3, 5, 7 - PR10 & & \\
\hline \multicolumn{4}{|l|}{$\begin{array}{c}\text { Producción, transporte, } \\
\text { almacenamiento y uso ade- } \\
\text { cuado de productos químicos }\end{array}$} \\
\hline $\begin{array}{c}\text { Gestión adecuada de riesgos } \\
\text { a nivel político, técnico } \\
\text { y de comunidad }\end{array}$ & Objetivos 1, 2, 3 & $\mathrm{D} 2$ & Objetivo 13 \\
\hline \multicolumn{4}{|l|}{$\begin{array}{c}\text { Vigilancia y control de } \\
\text { enfermedades transmisibles }\end{array}$} \\
\hline $\begin{array}{c}\text { Conocimiento sobre el cambio } \\
\text { y la variabilidad climática }\end{array}$ & & Objetivos 1, 2, 3 - D3 & Objetivo 13 \\
\hline
\end{tabular}

Tabla 6. Matriz de intervención sobre los determinantes del riesgo de desastres

A través de esta herramienta se observa claramente que la mayor incidencia sobre los factores subyacentes del riesgo de desastre proviene de la agenda de desarrollo sostenible. Contrariamente, la agenda de clima es la que menos incide en los factores. Es interesante observar que, aun cuando la agenda de reducción del riesgo menciona explícitamente la relevancia de los factores subyacentes del riesgo de desastre y la necesidad de abordarlos, a la hora de incidir con intervenciones específicas sus propuestas son escasas o insuficientes, dependiendo en buena manera de la agenda de desarrollo para llevarlo a cabo.

Conviene en este momento reflexionar sobre la superposición que hoy en día se confiere a la RDD y la adaptación, particularmente cuando se habla de la variabilidad climática y los extremos climáticos, incluyendo gestión de los riesgos relacionados con el cambio climático. Sin embargo, conviene profundizar un poco más. Mientras la reducción del riesgo enfatiza la disminución de la probabilidad de que una amenaza se traduzca en un desastre, considerando las características de lo expuesto y los impactos causados, la adaptación consiste en hacer ajustes para incorporar cambios, incluyendo nuevos riesgos, en el continuo devenir, para evitar o minimizar el daño que los cambios climáticos y los fenómenos meteorológicos extremos pueden causar. Pareciera entonces que la adaptación es una idea mayor que la reducción del riesgo ya que contiene a esta última y además acepta, convive y se transforma con y por el cambio que está ocurriendo (Schipper, 2014).

\subsection{Una mirada a los indicadores de la Agenda de Desarrollo Sostenible}

Considerando la trascendencia de la agenda de desarrollo sostenible en los factores subyacentes del riesgo de desastre se decidió hacer una búsqueda de referencias explícitas al tema del riesgo en los indicadores propuestos, encontrando una correspondencia entre cuatro indicadores de la agenda de desarrollo e indicadores propuestos para la implementación del Marco de Sendai (Maskrey, 2016). Estos indicadores pertenecen a los ODS 1, 11 y 13 como lo ilustra la Tabla 7. 
Correspondencia Entre Indicadores de Sostenibilidad y de Reducción del Riesgo

\begin{tabular}{l|l|l}
\multicolumn{2}{c}{ Correspondencia Entre Indicadores de Sostenibilidad y de Reducción del Riesgo } \\
\hline \multicolumn{1}{c|}{ ODS } & \multicolumn{1}{c|}{ ODS - Indicador } & \multicolumn{1}{c}{ MSRRD - Indicador } \\
\hline $\begin{array}{l}\text { 1. Poner fin a la pobreza en todas } \\
\text { Susmas en todo el mundo }\end{array}$ & $\begin{array}{l}1.5 \text { Para } 2030 \text { fortalecer la resiliencia } \\
\text { de los pobres y los que están en } \\
\text { situaciones vulnerables y reducir su } \\
\text { exposición y vulnerabilidad a eventos } \\
\text { extremos relacionados con el clima y } \\
\text { otros choques económicos, sociales y } \\
\text { ambientales y desastres }\end{array}$ & $\begin{array}{l}\text { - Número de muertes, personas des- } \\
\text { aparecidas y personas afectadas por } \\
\text { desastres por cada } 100.000 \text { personas } \\
\text { directos en relación con el producto } \\
\text { interno bruto (PIB) mundial } \\
\text { - Número de países con estrategias } \\
\text { de RRD nacionales y locales }\end{array}$ \\
\hline
\end{tabular}
asentamientos humanos sean inclusivos, seguros, resilientes y sostenibles
11. Lograr que las ciudades y los

11.5 Para el año 2030, reducir significativamente el número de muertes y el número de personas afectadas y disminuir sustancialmente las pérdidas económicas directas en relación con el PIB mundial causadas por desastres, incluidos los desastres relacionados con el agua, en las situaciones de vulnerabilidad

11.b En 2020, aumentar sustancialmente el número de ciudades y asentamientos humanos que adopten y apliquen políticas y planes integrados para la inclusión, la eficiencia de los recursos, la mitigación y la adaptación al cambio climático, la resiliencia ante los desastres y desarrollar y aplicar, con el Marco de Sendai para la RRD, la gestión holística del riesgo de desastres a todos los niveles

13. Adoptar medidas urgentes para combatir el cambio climático y sus efectos
13.1 Fortalecer la capacidad de adaptación y capacidad de adaptación a los peligros relacionados con el clima y los desastres naturales en todos los países
- Número de muertes, personas desaparecidas y personas afectadas por desastres por 100.000 personas - Pérdidas económicas de desastres directos en relación con el PIB mundial, servicios básicos

- Número de países con estrategias nacionales y locales de reducción de riesgos

- Proporción de gobiernos locales que adoptan y aplican estrategias locales de RRD en consonancia con el Marco Sendai
- Número de muertes, personas desaparecidas y personas afectadas por desastres por 100.000 habitantes - Estrategias nacionales y locales de RRD

- Proporción de gobiernos locales que adopten y apliquen estrategias locales de RRD en consonancia con el Marco Sendai

\section{CONCLUSIONES}

La Agenda Pos-2015 compuesta a su vez por las agendas de reducción del riesgo de desastre, de clima y de desarrollo sostenible constituyen un acuerdo sobre el desarrollo a gran escala, que incluye la mitigación, la adaptación y las finanzas asociadas al cambio climático, y el Marco Sendai para la gestión del riesgo de desastres.

Los resultados alcanzados a través de las herramientas empleadas en este estudio evidencian que la agenda de reducción del riesgo hace un fuerte énfasis en la gestión integrada y anticipada de los riesgos, pasando de la gestión de eventos a la gestión de los procesos que generan riesgos. Igualmente queda claro que la gestión del riesgo de desastres no debe considerarse como un "sector" en sí mismo, sino una práctica que permea todos los sectores, que busca evitar la construcción de riesgos, reducir los riesgos existentes y fortalecer la resiliencia. Desde otra perspectiva, el verdadero desarrollo implica necesariamente su sostenibilidad, por ello es incompatible con la existencia de riesgos sin gestionar ya que puede comprometer los logros obtenidos.

El análisis de la Agenda Pos-2015 llevado a cabo desde la perspectiva de los determinantes del riesgo de desastres recae mayormente en la agenda de desarrollo, a través de múltiples dimensiones, hecho que contrasta con las agendas de reducción del riesgo y la de clima, las cuales se ubican más en un rol de abogacía. Este hallazgo confirma la tesis de Hagman (1984) acerca de que los desastres, en este caso el riesgo de desastres, son el resultado de problemas no resueltos del desarrollo. 
En la búsqueda de contrarrestar la construcción del riesgo se identificaron claramente cuatro dominios principales y un nodo crítico, que marcan una trayectoria de cambio y que deben ser objeto de intervención. Los dominios son: 1) ocupación segura del territorio; 2) medios de vida suficientes y resilientes; 3 ) ecosistemas robustos y resilientes; y 4) manejo adecuado de emergencias; el nodo crítico está representado por la erradicación de la pobreza absoluta.

Es indispensable que exista una coherencia, complementariedad e interdependencia entre las agendas analizadas en este estudio, de forma de trascender el anhelado crecimiento económico para apuntalar un verdadero desarrollo sostenible, incidiendo sobre los factores subyacentes del riesgo de desastre.

\section{REFERENCIAS}

Acosta, V. (2005). El riesgo como construcción social y la construcción social de riesgos. Desacatos, (19), 11-24.

Blaikie, P. M., Cannon, T., Davies, I., \& Wisner, B. (Eds.). (1994). At risk: natural hazards, people’s vulnerability, and disasters. London \& New York: Routledge.

C2E2. (2015). Outcomes of The U.N. Climate Change Conference In Paris. The Center for Climate and Energy Solutions (C2ES). Recuperado el 4 de Abril de 2017, del sitio web del C2E2: https://www.c2es.org/docUploads/cop-21-paris-summary-02-2016-final.pdf

Corbyn, C. (2016). The Paris Agreement on climate change - A summary. Recuperado el 4 de Abril de 2017, del sitio web del National Assembly for Wales Research Service: https://assemblyinbrief.wordpress.com/2016/04/04/the-paris-agreement-on-climate-change-a-summary/

Goldhagen, J., \& Mercer, R. (2010). A Child Rights and Equity-Based Assessment of the Impact of Global Climate Change on Children's Health and Well-Being. International Public Health Journal, 2 (4), 541-551.

Hagman, G. (1984). Prevention better than cure: report on human and environmental disasters in the Third World. (3rd ed.). Stockholm: Swedish Red Cross.

Maskrey, A. (2016). Monitoring the Sendai Framework for DRR 2015-2030 and promoting coherence with the SDGs. Recuperado el 1 de Abril de 2017, del sitio web del IISD: http://sdg.iisd.org/news/ experts-discuss-aligning-indicators-for-sdgs-and-sendai-framework-for-drr/

McGaghie, W. C., Bordage, G., \& Shea, J. A. (2001). Manuscript Introduction: Problem Statement, Conceptual Framework, and Research Question. Academic Medicine, 76(9), 923-924.

Naciones Unidas, A. G. Desarrollo sostenible: reducción del riesgo de desastres, Pub. L. No. A/71/644, 39 (2016). Recuperado el 30 de Marzo de 2017, del sitio web Prevention Web http://www.preventionweb.net/files/50683_oiewgreportspanish.pdf

Naciones Unidas - ISDR. (2009). Terminología sobre. Reducción del Riesgo de Desastres. Génova: Naciones Unidas. Recuperado el 30 de Marzo de 2017, del sitio web de Naciones Unidas: http://www.unisdr.org/files/7817_UNISDRTerminologySpanish.pdf

Roberts, E., Andrei, S., Huq, S., \& Flint, L. (2015). Resilience synergies in the post-2015 development agenda. Nature Climate Change, 5(12), 1024-1025.

Sarmiento, J. P. (2017). Chapter 28. International Organisations Doing Disaster Risk Reduction Including Climate Change Adaptation. In The Routledge Handbook of Disaster Risk Reduction Including Climate Change Adaptation (First, p. 515). Abingdon, Oxon \& New York, NY: Routledge.

Schipper, L. (2014). Disasters, climate change and development: Reducing risk by tackling the drivers of vulnerability. Recuperado el 1 de Abril de 2017, del sitio web del Stockholm Environment Institute: https://www.sei-international.org/mediamanager/documents/Publications/Climate/SEI-DB-2014Climate-change-DRR-LR.pdf 
TANGO International. (2012). Livelihoods and Disaster Risk Reduction. Miami, FL: Florida International University.

UNISDR. (2015). Reading the Sendai Framework for Disaster Risk Reduction 2015 - 2030. Recuperado el 1 de Abril de 2017, del sitio web de Naciones Unidas:

http://www.unisdr.org/files/46694_readingsendaiframeworkfordisasterri.pdf

United Nations, F. C. on C. C. Adoption of The Paris Agreement, Pub. L. No. FCCC/CP/2015/L.9, 40 (2015). Recuperado el 1 de Abril de 2017, del sitio web de United Nations:

https://unfccc.int/resource/docs/2015/cop21/eng/lo9.pdf

Weiss, C. H. (1998). Nothing as Practical as Good Theory: Exploring Theory-based Evaluation for Comprehensive Community Initiatives for Children and Families. In J. P. Connell (Ed.),

New Approaches to Evaluating Community Initiatives: Concepts, Methods, and Contexts (pp. 65-92). Washington, DC: Aspen Institute.

Zalta, E. N. (2009). Logic and Ontology. In Stanford Encyclopedia of Philosophy (Spring 2009 Edition). Stanford, CA: Stanford University. Recuperado el 1 de Abril de 2017, del sitio web de Stanford University: https://plato.stanford.edu/archives/spr2oog/entries/logic-ontology/\#3.1 